\title{
A method for the assessment of the optimal parameter of discrete-time switch model
}

\author{
R. Razzaghi*, C. Foti, M. Paolone, F. Rachidi \\ École Polytechnique Fédérale de Lausanne, 1015 Lausanne, Switzerland
}

\section{A R T I C L E I N F O}

\section{Article history:}

Received 15 November 2013

Received in revised form 13 January 2014

Accepted 10 February 2014

Available online 16 March 2014

\section{Keywords:}

Discrete-time switch model

Modified nodal analysis

Fixed admittance matrix nodal method

Real-time simulations

\begin{abstract}
A B S T R A C T
This paper proposes a novel method for the optimal parameter selection of the discrete-time switch model used in circuit solvers that adopt the fixed admittance matrix nodal method (FAMNM) approach. As known, FAMNM-based circuit solvers allow to reach efficient computation times, in particular for realtime simulation applications, since they do not need the inversion of the circuit nodal admittance matrix. However, these solvers need to optimally tune the so-called discrete switch conductance, since this parameter might largely affect the simulations accuracy. Within this context, we propose a method for the determination of the discrete-time switch conductance which is obtained by minimizing the distance between the eigenvalues of the original circuit's nodal admittance matrix with those associated with the circuit including the discrete-time switches. The method is proven to provide values of the discrete-time switch conductance that maximize the simulation accuracy and minimize the losses on this artificially introduced parameter. Additionally, the proposed method avoids the use of trial-and-error process typically required when discrete-time switch conductances need to be addressed in FAMNM approach. The performances of the proposed method are demonstrated for circuits with single and multiple switches in which passive RLC elements and transmission lines are both considered.
\end{abstract}

(c) 2014 Elsevier B.V. All rights reserved.

\section{Introduction}

Accurate and computationally efficient time-domain simulation of power systems including switches (such as traditional circuit breakers or power electronic devices), is a challenging subject since the tradeoff between accuracy and computation time depends on the adopted models of the switching devices, especially when realtime constraints need to be achieved.

Detailed switch models reproducing their physical properties are used when studying phenomena such as switching losses, arcing times and electromagnetic transients associated with switching-arc extinction. However, in many power systems applications, these sophisticated models cannot be used because of their required computational efforts and complexity of implementation. As a result, simplified switch models have been proposed in the literature (e.g. [1,2]).

One of the most popular methods consists in representing switches as lumped electrical components. The simplest approach is the so-called two-valued resistor model where two resistors, characterized by large differences of their resistance values, are

\footnotetext{
* Corresponding author. Tel.: +41 216934815; fax: +41 216934662

E-mail address: reza.razzaghi@epfl.ch (R. Razzaghi).
}

associated with each state of the switch. The typical representation consists in replacing the switch by a resistor characterized by a "small" value of resistance for the "closed-state" and a "large" value for the "open-state". However, in this case the system's admittance matrix needs to be updated and re-factorized after each switching state change (e.g. $[3,4]$ ).

Within the context of real-time simulations, updating the admittance matrix imposes additional computational burden to the solution algorithms that need to be executed within a determined time window. As a consequence, the admittance matrix re-factorization represents a major obstacle to satisfy the computational constraints.

A possible approach to circumvent this problem is the use of modeling techniques that keep the system admittance matrix constant (e.g., $[5,6])$. To this end, discrete circuit models for switching devices were proposed in $[1,7,8]$. The basic idea is that the switch could be represented by a relatively small inductance when its state is 'closed' and by a relatively small capacitance when its state is 'open'. As a consequence, the discrete-time switch model is represented by an equivalent conductance $\left(G_{s}\right)$ in parallel with a current source controlled by the so-called history term (e.g., [4]). The consequence of such a representation is an approach called fixed admittance matrix nodal method (FAMNM) [9]. In the FAMNM, the discrete-time switch conductance $G_{s}$ is kept constant during 
switches state-transitions, the change of the switches state affects only the value of the current source which does not appear in the circuit admittance matrix. On the other hand, such a switch representation introduces artificial transients $[2,5]$.

Solutions to solve this problem have been proposed in the literature. In particular, a damping resistance can be added in series to the discrete-time switch model [10]. However, this approach poses the problem of the optimal choice for the value of such a damping resistance as well as the inclusion of artificial losses into the system.

A different approach to solve this problem is the optimal selection of the $G_{s}$ parameter. One possibility is to consider an a priori value for $G_{s}$ and, then, find the corresponding optimal value by comparing the simulation results with benchmark ones in order to minimize the relevant errors [2] (i.e., obtained by off-line simulations where the switches are represented by ideal devices). However, such a trial-and-error approach provides solutions that require specific and time consuming assessments in which the uniqueness of the solution is not guaranteed.

The authors of this paper have presented a preliminary analysis in [11] on the possibility of finding the optimal discrete-time switch $G_{s}$ conductance by solving a suitable optimization problem. The current paper aims at discussing more in detail the method presented in [11], and providing its extension to the case of systems with multiple switches. Specifically, the proposed method is based on the minimization of the Euclidian distance between the eigenvalues of the FAMNM-based network admittance matrix and those associated with the admittance matrices of the reference networks corresponding to all possible switches permutations where the switches are represented by ideal ones. It is worth noting that, compared to the existing methods that rely on the extensive and numerous trial-and-error simulation studies, the proposed method allows to reduce the computations required to find the optimal discrete-time switch $G_{S}$ conductance.

The structure of the paper is as follows: Section 2 summarizes the formulation of the FAMNM. Section 3 describes the problem definition. Section 4 presents the proposed method to find the optimum values for the conductance of the discrete-time switches generalized to the case of systems with multiple switches. Section 5 presents the validation of the proposed method by making reference to three examples. Finally, Section 6 concludes the paper with the final remarks and potential implementation of what discussed in the paper.

\section{FAMNM representation of the switch}

The idea of FAMNM is the discrete-time representation of the switch with a constant impedance model [1,7-9]. Such an approach assumes that the equivalent model of the ideal switch is piecewise linear and could be represented by a capacitance when it is open and an inductance when it is closed. The inductance and capacitance are represented, in a discrete form, by a conductance in parallel with a current source. In order to set the value of the conductance for both switch states, in case the backward Euler numerical integration method is used, the following constraint should be satisfied:

$G_{s}=\frac{C_{s}}{\Delta t}=\frac{\Delta t}{L_{s}}$

where $C_{S}$ and $L_{S}$ are the discrete-time switch capacitance and inductance respectively, and $\Delta t$ is the simulation time-step. For other numerical integration methods, a similar approach could be applied to relate $G_{s}$ to the switch capacitance and inductance. It is worth observing that Eq. (1), with different analytical forms, holds also in case other numerical integration techniques are used [1].

As a consequence of this representation, the relevant model is composed of a constant conductance in parallel with a current

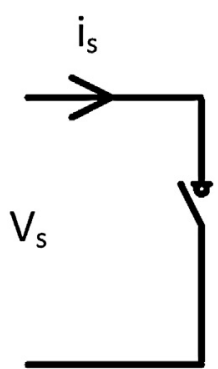

a

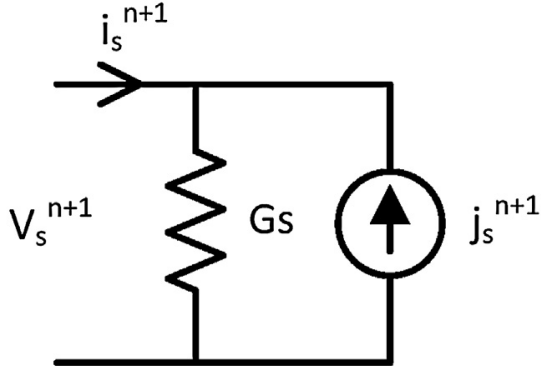

b
Fig. 1. Ideal switch (a) and corresponding discrete-time model (b). Source: Adapted from [1].

source (see Fig. 1). As a function of the switch on/off state, the value of the current source $\left(j_{s}^{n+1}\right.$ in Fig. 1$)$ is updated at each time-step based on the switch current/voltage. The advantage of this method is that the value for the switch conductance $G_{s}$ is fixed irrespective of the switch on/off state. As a result, the nodal admittance matrix will remain unchanged during switching operations as the switch state only affects the value of the shunt current source. The current source associated with the switch at the simulation step $n+1$ is defined as [1]:

$J_{s}^{n+1}\left\{\begin{array}{cc}-i_{s}^{n} & \text { for the 'on' state } \\ G_{s} v_{s}^{n} & \text { for the 'off' state }\end{array}\right.$

According to [1], one approach for determining the value of $G_{S}$ is to select $C_{S}$ and $L_{S}$ equal to the corresponding real switch parameters. Then, the values of $G_{s}$ and $\Delta t$ could be determined using (1). However, the main drawback of this approach is that the required simulation time step might become extremely small resulting in prohibitive computational times.

As already stated in the introduction, a different procedure refers to the assessment of an optimal $G_{s}$ value by means of a trialand-error process where benchmark results are obtained by means of off-line simulations carried out by adopting ideal switches. However, such an approach requires non-negligible pre-computational efforts.

\section{Discrete-time representation of electrical systems}

Generally, there are two main types of solution methods currently used in the field of power system, power electronics and electronic circuit simulations [12]: (i) modified nodal analysis (MNA) and (ii) state-space (SS) approach. In this study, in order to formulate the network equations, MNA has been selected similarly to [1]. This choice is supported by the fact that the MNA provides a straightforward way of directly integrating the above illustrated discrete-time switch model.

As known, the MNA formulation is expressed as follows:

$\left[A_{n}\right]\left[x_{n}\right]=\left[b_{n}\right]$

where matrix $\left[A_{n}\right]$, in the discrete-time domain, is formed by the discrete representation of the network elements; $\left[x_{n}\right]$ is the vector of unknown network's node voltages and branch currents; and $\left[b_{n}\right]$ is a vector composed of the independent sources and current history terms related to the network components. At each iteration, the unknown vector $\left[x_{n}\right]$ is calculated and, then, the vector $\left[b_{n}\right]$ is updated. As already mentioned, representing switches with FAMNM allows to keep $\left[A_{n}\right]$ fixed during switching transitions.

In order to solve Eq. (3) in discrete-time, a suitable numerical integration method should be used. Among the several methods available, backward Euler and the trapezoidal methods are the 


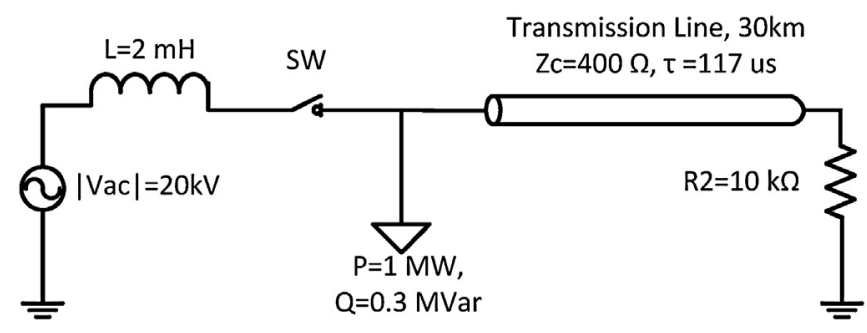

Fig. 2. Schematic representation of the test case composed of a single-conductor transmission line and a switch.

most common in power system applications [13]. Together with these traditional methods, new numerical integration techniques based on midpoint methods have been proposed. An example of these techniques is the two-stage diagonally implicit Runge-Kutta method presented in [14].

In this paper, the backward Euler method has been adopted. As it will be clarified next, the validation of the proposed method has been done using the EMTP-RV simulation environment $[15,16]$ where the backward Euler integration method was used. In this respect, it is worth observing that the proposed approach is independent from the adopted numerical integration technique. Indeed, it relies on the FAMNM modeling approach of electrical circuits. The use of a different numerical integration techniques (i.e., backward Euler and the trapezoidal methods) will only change the elements that appear in the discrete-time network admittance matrix $A_{n}$ of (3). The backward-Euler integration technique has been selected since it has been largely used in the literature (e.g., $[1,5,9])$ to formulate the FAMNM approach.

In view of the use of the MNA, all the network components should be discretized to form the so-called nodal equations $[17,18]$. Lumped passive elements $(R, L, C)$ are represented by their discrete companion models composed of an equivalent conductance in parallel with a current source representing the history terms $[18,19]$. The values of the equivalent conductance and the current source are determined based on the applied numerical integration method. Concerning the case of transmission lines, one of the most popular modeling approach is the so-called Bergeron method [20]. It allows a straightforward representation of constant (frequency-independent) transmission line models [4] and, with some adaptations, it can also be applied to the case of frequencydependent transmission lines [21]. As well known, this approach is based on a circuit representation of the telegraphers' equations where each line termination is replaced by means of a lumped impedance in parallel with a controlled current or voltage source.

\subsection{Problem definition}

In order to illustrate this problem, the time-domain simulation of the inrush of a single-conductor transmission line is considered. As it is shown in Fig. 2, the network is composed of an ideal source, representing an infinite power bus, supplying a HV/MV transformer that feeds a $1 \mathrm{MW} / 0.3 \mathrm{MV}$ ar load and a transmission line. The transmission line parameters are those of a typical $20 \mathrm{kV}$ lossy overhead line, namely a surge impedance of $400 \mathrm{Ohms}$ and a propagation time equal to $117 \mu \mathrm{s}$. In order to simplify the interpretation of the results, a single-phase model has been considered.

This network has been simulated in EMTP-RV simulation environment where the switch was modeled using either an ideal switch (reference case), or a discrete-time model with different values for the conductance. Fig. 3 shows the effect of the switch $G_{s}$ value on the accuracy of the current at the feeding terminal of the transmission line. It can clearly be observed that the adopted value

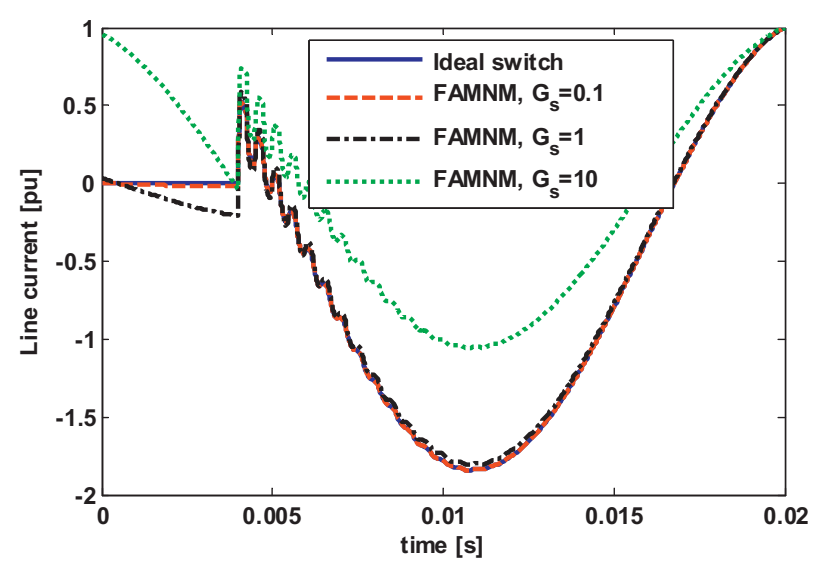

Fig. 3. Line current at the beginning of the line for four switch representation models (i) ideal switch, (ii) FAMNM representation for $G_{s}=0.1$, and (iii) FAMNM representation for $G_{s}=1$, and (iv) FAMNM representation for $G_{s}=10$.

for $G_{s}$ affects dramatically the simulation accuracy of the FAMNM vs. the reference one in which the ideal switch has been considered.

\section{Proposed method for the optimal evaluation of discrete-time switches conductance}

The proposed method is based on the minimization of the Euclidian distance between the eigenvalues of the network admittance matrix $\left[A_{n}\right]$ based on FAMNM, and those associated with the admittance matrices of reference networks corresponding to the all possible switching permutations. In order to clearly describe the proposed method, it will be first explained for the case of a network with a single switch. Then, it is generalized for the case of networks with multiple switches.

\subsection{Proposed method for networks composed of a single switch}

The proposed method allows for the evaluation of the optimal value of the switch conductance $G_{s}$ as the solution of an optimization problem where the objective function is associated with the distances between the $\left[A_{n}\right]$ eigenvalues obtained using the FAMNM (of course, this set of eigenvalues is inherently function of $G_{S}$ ) and two other sets of eigenvalues of matrices $\left[A_{n}\right]$ related to the 'on' and 'off' states of the considered switch. In this respect, it is worth observing that the proposed approach is a formal process aiming at identifying the characteristics of the discrete-time switch conductance that minimizes a suitably defined objective function that exhibits minimum coinciding with those of objective functions related to artificial losses introduced by the FAMNM or to the errors in the reference simulated waveforms, see Section 4.3 for further details.

Let $\left[A_{n}^{p}\right],\left[A_{n}^{c}\right]$, and $\left[A_{n}^{o}\right]$ be the nodal admittance matrices when the switch is represented by FAMNM, ideal switch in 'on' state, and ideal switch in 'off' state, respectively. The three matrices are all supposed to be of rank $n$. As stated in a fundamental theorem of linear algebra, if $\mathbf{B}$ is a base of the vector space $\mathbf{V}$ (of dimension $n$ ) and $\mathbf{W}$ another vector space of dimension $n$, for each transformation $\varphi: \mathbf{B} \rightarrow \mathbf{W}$, there is one and only one linear map $\mathbf{T}: \mathbf{V} \rightarrow \mathbf{W}$ such that $\left.\boldsymbol{T}\right|_{\boldsymbol{B}}=\varphi$. Now, since $\left[A_{n}^{p}\right],\left[A_{n}^{c}\right]$, and $\left[A_{n}^{o}\right]$ are real matrices of rank $n$, we can define three bases $\mathbf{B}_{p}, \mathbf{B}_{c}$ and $\mathbf{B}_{o}$ of $R^{n}$ associated with the respective eigenvalues:

$$
\begin{aligned}
& B_{p} \leftrightarrow \lambda_{i}^{p}\left(G_{s}\right)=\operatorname{eig}\left\{\left[A_{n}^{p}\right]\right\}, \quad i=1,2, \ldots, n \\
& B_{c} \leftrightarrow \lambda_{i}^{c}=\operatorname{eig}\left\{\left[A_{n}^{c}\right]\right\}, \quad i=1,2, \ldots, n \\
& B_{o} \leftrightarrow \lambda_{i}^{o}=\operatorname{eig}\left\{\left[A_{n}^{o}\right]\right\}, \quad i=1,2, \ldots, n
\end{aligned}
$$




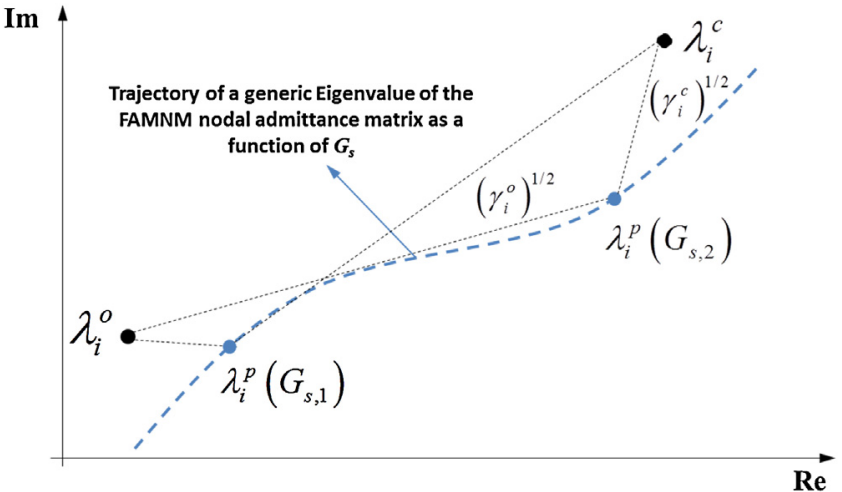

Fig. 4. Definition of Euclidian distance between eigenvalues of the network admittance matrix based on FAMNM and those associated with the admittance matrices of two reference networks.

Note that $\lambda_{i}^{p}\left(G_{s}\right), \lambda_{i}^{c}, \lambda_{i}^{o}$ denote respectively the corresponding eigenvalues for each nodal admittance matrix. As already clarified, $\lambda_{i}^{p}\left(G_{s}\right)$ is a function of $G_{s}$ whereas, $\lambda_{i}^{c}$ and $\lambda_{i}^{o}$ are fixed.

Since to each matrix $\left[A_{n}^{p}\right],\left[A_{n}^{c}\right]$, and $\left[A_{n}^{o}\right]$ we can associate a unique base ( $\mathbf{B}_{p}, \mathbf{B}_{c}$ and $\mathbf{B}_{o}$ respectively), in view of the fundamental theorem of linear algebra, these transformations are unique. Therefore, the objective is to determine $\mathbf{B}_{p}$ such that $\left.\boldsymbol{T}\right|_{\boldsymbol{B} p}$ provides results that are as close as possible to both $\left.\boldsymbol{T}\right|_{\boldsymbol{B} c}$ and $\left.\boldsymbol{T}\right|_{\boldsymbol{B} 0}$. A possible metric to achieve such a property is to minimize the squared Euclidian distances for each eigenvalue calculated as:

$\gamma_{i}^{c}\left(G_{s}\right)=\left\{\operatorname{Re}\left[\lambda_{i}^{p}\left(G_{s}\right)\right]-\operatorname{Re}\left[\lambda_{i}^{c}\right]\right\}^{2}+\left\{\operatorname{Im}\left[\lambda_{i}^{p}\left(G_{s}\right)\right]-\operatorname{Im}\left[\lambda_{i}^{c}\right]\right\}^{2}$

$\gamma_{i}^{o}\left(G_{s}\right)=\left\{\operatorname{Re}\left[\lambda_{i}^{p}\left(G_{s}\right)\right]-\operatorname{Re}\left[\lambda_{i}^{o}\right]\right\}^{2}+\left\{\operatorname{Im}\left[\lambda_{i}^{p}\left(G_{S}\right)\right]-\operatorname{Im}\left[\lambda_{i}^{o}\right]\right\}^{2}$

In these equations, $\gamma_{i}^{c}\left(G_{s}\right)$ and $\gamma_{i}^{o}\left(G_{s}\right)$ denote, as a function of $G_{S}$, the squared Euclidian distances between the $i$ th eigenvalue of $\left[A_{n}^{p}\right]$ and the corresponding one of $\left[A_{n}^{c}\right]$ and $\left[A_{n}^{o}\right]$, respectively. These distances, for a generic $i$ th eigenvalue are illustrated in Fig. 4.

Then, the following total distance (for a given eigenvalue) can be defined:

$\eta_{i}\left(G_{S}\right)=\gamma_{i}^{o}\left(G_{s}\right)+\gamma_{i}^{c}\left(G_{s}\right)$

It is possible to define an objective function as the sum of all normalized distances:

$\Gamma\left(G_{s}\right)=\sum_{i=1}^{n}\left(\frac{\eta_{i}\left(G_{s}\right)}{\max \left\{\eta_{i}\left(G_{s}\right)\right\}}\right)$

Note that the normalization is done in order to give equal weight to all eigenvalues distances. The optimum value for $G_{s}$ is defined as the one that minimizes the objective function (10). In other words:

$G_{s}^{*}=\left.\arg \right|_{G_{S}} \min \left\{\left(G_{S}\right)\right\}$

\subsection{Extension for networks with multiple switches}

For the case of a network with $N$ switches, the number of possible switching permutations is $2^{N}$. Therefore, there are $2^{N}$ set of eigenvalues of the nodal admittance matrix associated with ideal switches representations, namely:

$\lambda_{i}^{x}=\operatorname{eig}\left\{\left[A_{n}^{x}\right]\right\}, \quad\left\{\begin{array}{c}i=1,2, \ldots, n \\ x \in 2^{N}\end{array}\right.$

where $x$ is one of the possible switches permutations. Additionally, the eigenvalues of nodal admittance matrix associated with

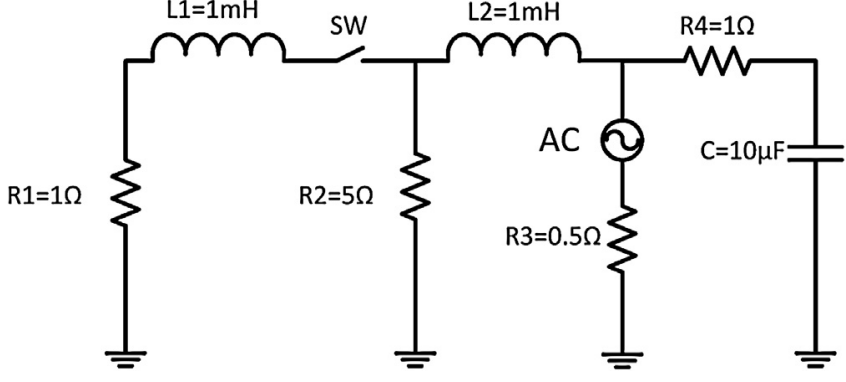

Fig. 5. Schematic representation of the RLC case study including one switch.

the FAMNM switch representation are function of switches conductances, namely:

$\lambda_{i}^{p}\left(G_{s 1}, G_{s 2}, \ldots\right)=\operatorname{eig}\left\{\left[A_{n}^{p}\right]\right\}, \quad i=1,2, \ldots, n$

where, for each switch, a different conductance value has been considered.

The squared Euclidian distances associated with the $i$ th eigenvalue have to be calculated for all possible permutations:

$$
\begin{aligned}
& \eta_{i}\left(G_{s 1}, G_{s 2}, \ldots\right)=\sum_{x}\left(\left\{\operatorname{Re}\left[\lambda_{i}^{p}\left(G_{s 1}, G_{s 2}, \ldots\right)\right]-\operatorname{Re}\left[\lambda_{i}^{x}\right]\right\}^{2}\right. \\
& \left.\quad+\left\{\operatorname{Im}\left[\lambda_{i}^{p}\left(G_{s 1}, G_{s 2}, \ldots\right)\right]-\operatorname{Im}\left[\lambda_{i}^{x}\right]\right\}^{2}\right)
\end{aligned}
$$

The objective function extended to the general case reads:

$\Gamma\left(G_{s 1}, G_{s 2}, \ldots\right)=\sum_{i=1}^{n}\left(\frac{\eta_{i}\left(G_{s 1}, G_{s 2}, \ldots\right)}{\max \left\{\eta_{i}\left(G_{s 1}, G_{s 2}, \ldots\right)\right\}}\right)$

The optimum values for the switches conductance come from the solution of the following optimal problem:

$G_{s 1}^{*}, G_{s 2}^{*}, \ldots=\left.\arg \right|_{G_{s 1}, G_{s 2}, \ldots} \min \left\{\Gamma\left(G_{s 1}, G_{s 2}, \ldots\right)\right\}$

It is worth observing that for networks with multiple switches, there might be preferred switching sequences. Thus, all the permutations are not used equally. Therefore, the number of the possible permutations and number of the switch conductances values can be significantly reduced.

\subsection{Method verification toward error and losses functions}

In order to verify the correctness of the solution provided by (16), we have first compared the objective function defined by (16) with an error function inferred from the differences between the voltage/current waveforms obtained for various values of $G_{s}$ and reference ones obtained using the ideal-switch model. Such an error function includes time-domain switch voltage and current waveforms subsequent to switch state transitions (in particular, subsequent to pairs of 'on'-'off' transitions). Indeed, as it is stated in [1], switch current error in 'off' state is proportional to $G_{s}$, whereas, switch voltage error in 'on' state is inversely proportional to $G_{s}$. This specific property has been exploited to define the error function. Specifically, the following procedure has been adopted: the switchcurrent error is calculated, for each of the $N_{1}$ switches in 'off' state, as the difference between the instantaneous values of the switch current given by the FAMNM solver and the current provided by a reference simulation where the switch is considered as an ideal device. The same procedure is considered to calculate the switch voltage error for each of the $N_{2}$ switches which are in 'on' state. For the $i$ th switch, the current and voltage errors, $E_{s w_{i}}^{I}\left(G_{s 1}, G_{s 2}, \ldots\right)$ and 


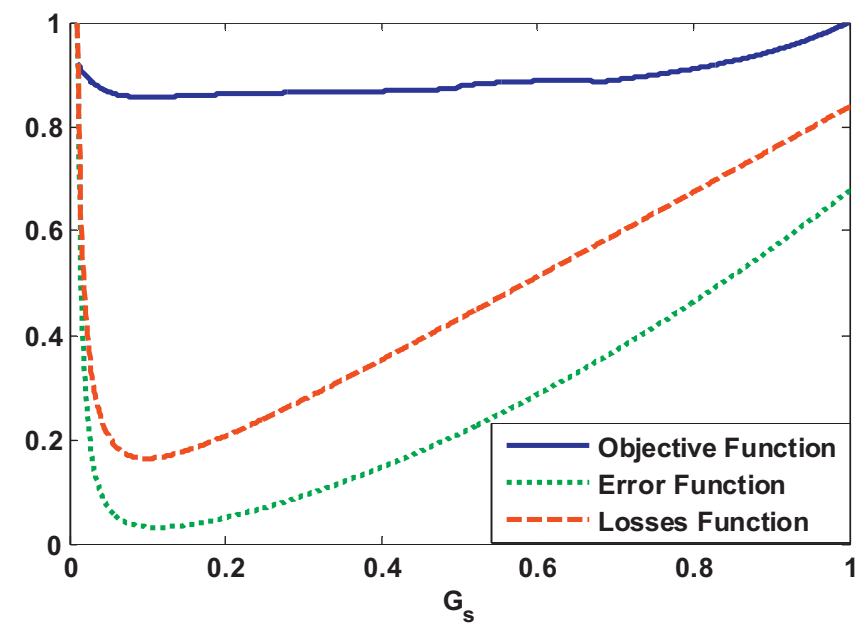

Fig. 6. Objective, error, and switch-losses functions for the case of the RLC test case of Fig. 5.

$E_{s w_{i}}^{V}\left(G_{s 1}, G_{s 2}, \ldots\right)$, are then given by:

$E_{\omega_{1}}^{I}\left(G_{s 1}, G_{s 2}, \ldots\right)=\sum_{k=0}^{m}\left[i_{\omega_{1}}\left(G_{s 1}, G_{s 2}, \ldots\right)_{k}-i_{k, \omega_{1}}^{*}\right]^{2}, \quad m=\frac{T}{\Delta t}$,

$\omega_{1}=1,2, \ldots, N_{1}$

$E_{\omega_{2}}^{V}\left(G_{s 1}, G_{s 2}, \ldots\right)=\sum_{k=0}^{m}\left[v_{\omega_{2}}\left(G_{s 1}, G_{s 2}, \ldots\right)_{k}-v_{k, \omega_{2}}^{*}\right]^{2}, \quad m=\frac{T}{\Delta t}$,

$\omega_{2}=1,2, \ldots, N_{2}$

Note that in (17) and (18), $T$ is a given time window where one possible switching permutation occurs. Discrete variables $i_{\omega_{1}}\left(G_{s 1}, G_{s 2}, \ldots\right)_{k}$ and $v_{\omega_{2}}\left(G_{s 1}, G_{s 2}, \ldots\right)_{k}$ correspond to the discretized instantaneous values of switch current and voltage when the switch is represented by its approximate model and, thus, they are function of all switches conductances. Discrete variables $i_{k, \omega_{1}}^{*}$ and $v_{k, \omega_{2}}^{*}$ are the corresponding discretized instantaneous switch current and voltage, obtained from reference simulations where the switch are represented as ideal devices.

To define the error function, the effect of all $N$ switches should be taken into account. Namely, for each possible permutation, the sum of the current errors for all switches in 'off' state and the sum of the voltage errors for all the switches in 'on' state are considered as:

$$
\begin{aligned}
& E^{\chi}\left(G_{s 1}, G_{s 2}, \ldots\right)=\sum_{\omega_{1}}\left(\frac{E_{\omega_{1}}^{I}\left(G_{s 1}, G_{s 2}, \ldots\right)}{\max \left(E_{\omega_{1}}^{I}\left(G_{s 1}, G_{s 2}, \ldots\right)\right)}\right) \\
& \quad+\sum_{\omega_{2}}\left(\frac{E_{\omega_{2}}^{V}\left(G_{s 1}, G_{s 2}, \ldots\right)}{\max \left(E_{\omega_{2}}^{V}\left(G_{s 1}, G_{s 2}, \ldots\right)\right)}\right)
\end{aligned}
$$

For all possible switching cases, the same procedure is applied by considering the same time window $T$ for the calculation and by changing the switches state.

Finally, in order to take into account all possible permutations, the overall error function is defined as the sum of normalized errors calculated for each possible permutation in (19), as follows:

$E\left(G_{s 1}, G_{s 2}, \ldots\right)=\sum_{x}\left(\frac{E^{x}\left(G_{s 1}, G_{s 2}, \ldots\right)}{\max \left(E^{x}\left(G_{s 1}, G_{s 2}, \ldots\right)\right)}\right)$

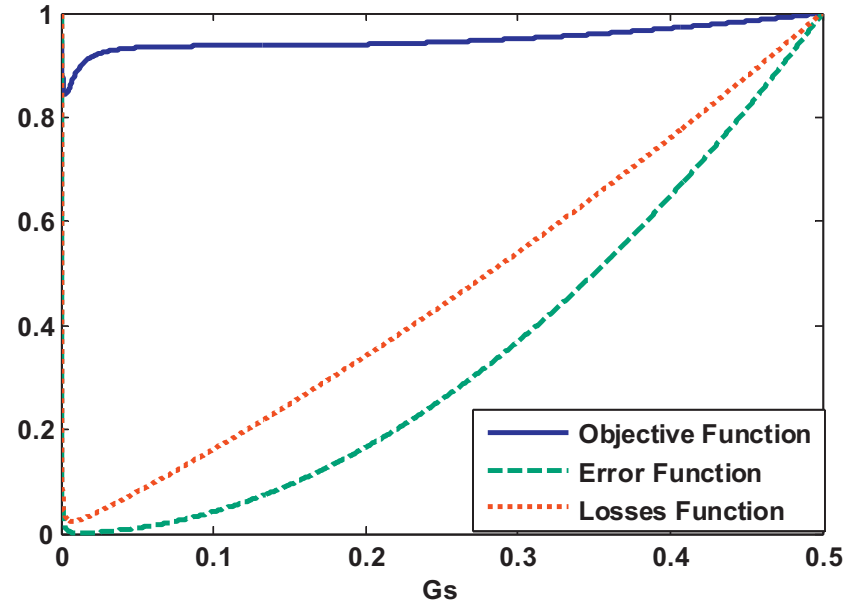

Fig. 7. Objective, error, and switch-losses functions for the case of transmission line test case (Fig. 2) with variable $G_{s}$.

A further way to verify the correctness of the solution provided by (16) is to compare it with another function that represents the switch losses when these devices are represented by using the FAMNM approach. For the case of an ideal switch, the switch losses are zero during the 'off' and 'on' states since switch current/voltage are null.

To this end, the switch losses in 'off' and 'on' states, $P_{\omega_{1}}^{O}\left(G_{s 1}, G_{s 2}, \ldots\right)$ and $P_{\omega_{2}}^{C}\left(G_{s 1}, G_{s 2}, \ldots\right)$ respectively, can be straightforwardly calculated as follows:

$$
\begin{gathered}
P_{\omega_{1}}^{O}\left(G_{s 1}, G_{s 2}, \ldots\right)=\frac{1}{m} \sum_{k=0}^{m}\left(v_{\omega_{1}}^{o}\left(G_{s 1}, G_{s 2}, \ldots\right)_{k} \cdot i_{\omega_{1}}^{o}\left(G_{s 1}, G_{s 2}, \ldots\right)_{k}\right), \\
m=\frac{T}{\Delta t}, \quad \omega_{1}=1,2, \ldots, N_{1} \\
P_{\omega 2}^{C}\left(G_{s 1}, G_{s 2}, \ldots\right)=\frac{1}{m} \sum_{k=0}^{m}\left(v_{\omega_{2}}^{c}\left(G_{s 1}, G_{s 2}, \ldots\right)_{k} \cdot i_{\omega_{2}}^{c}\left(G_{s 1}, G_{s 2}, \ldots\right)_{k}\right), \\
m=\frac{T}{\Delta t}, \quad \omega_{2}=1,2, \ldots, N_{2}
\end{gathered}
$$

where discrete variables $i_{\omega_{1}}^{O}\left(G_{s 1}, G_{s 2}, \ldots\right)_{k}, v_{\omega_{1}}^{o}\left(G_{s 1}, G_{s 2}, \ldots\right)_{k}$ and $i_{\omega_{2}}^{c}\left(G_{s 1}, G_{s 2}, \ldots\right)_{k}, v_{\omega_{2}}^{c}\left(G_{s 1}, G_{s 2}, \ldots\right)_{k}$ correspond to the discretized instantaneous values of switch current and voltage in 'off' and 'on' states respectively, when the switch is represented by the FAMNM.

The effect of all switches in a possible switching permutation is taken into account by considering the sum of all switches losses as:

$$
\begin{aligned}
& P^{x}\left(G_{s 1}, G_{s 2}, \ldots\right)=\sum_{\omega_{1}}\left(\frac{P_{\omega_{1}}^{O}\left(G_{s 1}, G_{s 2}, \ldots\right)}{\max \left(P_{\omega_{1}}^{O}\left(G_{s 1}, G_{s 2}, \ldots\right)\right)}\right) \\
& \quad+\sum_{\omega_{2}}\left(\frac{P_{\omega_{2}}^{C}\left(G_{s 1}, G_{s 2}, \ldots\right)}{\max \left(P_{\omega_{2}}^{C}\left(G_{s 1}, G_{s 2}, \ldots\right)\right)}\right)
\end{aligned}
$$

where $P^{x}\left(G_{s 1}, G_{s 2}, \ldots\right)$ is the overall loss for one of the possible switching permutation.

Then, the total losses function takes into account all possible switching permutations by summing the normalized overall losses calculated in (23):

$P\left(G_{s 1}, G_{s 2}, \ldots\right)=\sum_{x}\left(\frac{P^{x}\left(G_{s 1}, G_{s 2}, \ldots\right)}{\max \left(P^{x}\left(G_{s 1}, G_{s 2}, \ldots\right)\right)}\right)$ 


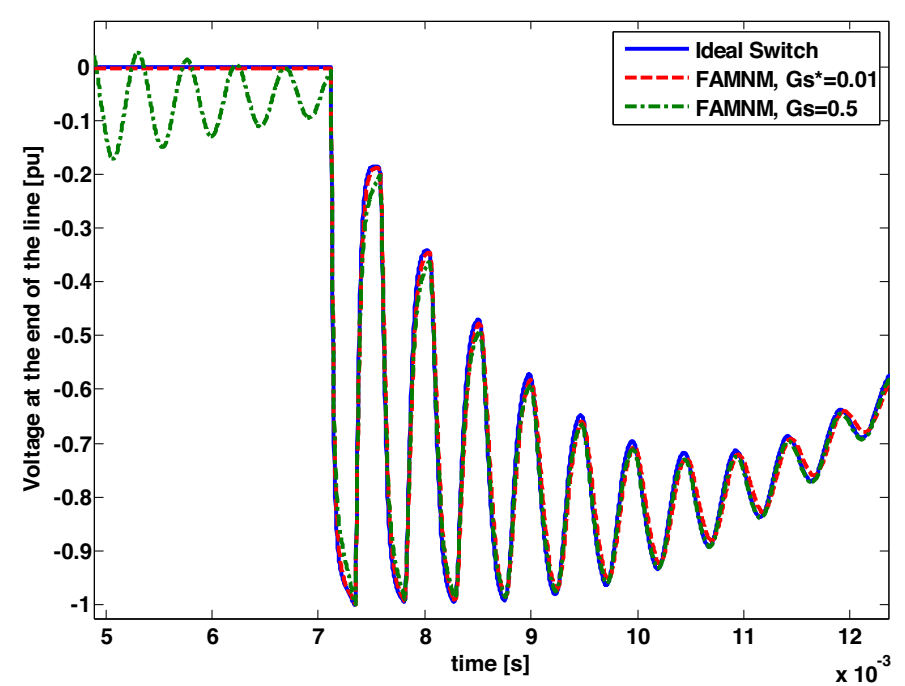

Fig. 8. Time-domain simulated waveforms for voltage at the end of the transmission line (second test case - Fig. 2) for different values of $G_{s}$.

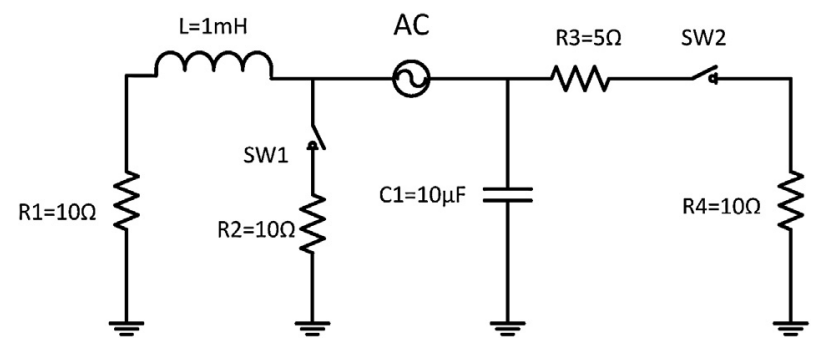

Fig. 9. Schematic representation of the RLC case study including two switches.

In the next section, we will show that the optimal $G_{s}$ value provided by (16) corresponds also to the minimum of the error function (20) and losses function (24), proving that the proposed approach satisfies these two criteria at the same time.

\section{Validation examples}

In order to validate our proposed method, two simulation cases are considered. The first simulation refers to electrical circuits composed of one switch. The second case study refers to electrical circuits composed of two switches. These case studies are carried out by making reference to two different types of electrical circuits: (i) circuits composed of RLC elements and (ii) circuits including transmission lines.

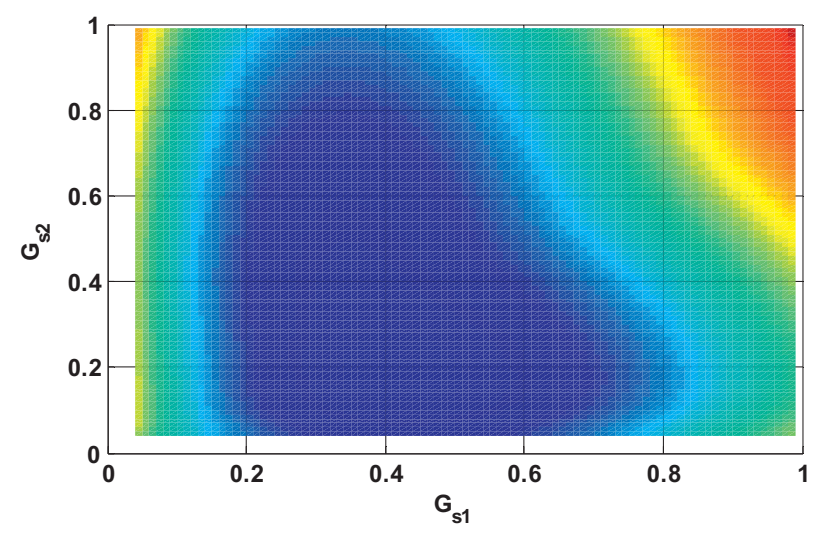

Fig. 10. Objective function used to assess the optimal $G_{s}$ values for the case of RLC circuit with two switches shown in Fig. 9.

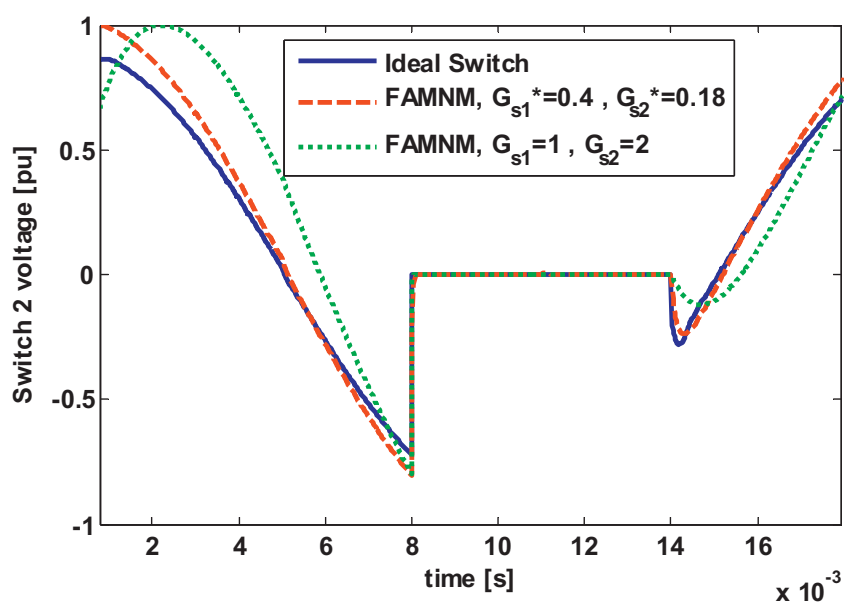

Fig. 11. Time-domain simulated waveforms for switch \#2 voltage for the optimal values of $G_{s 1}, G_{s 2}$ for the circuit composed of RLC elements and two switches.

\subsection{Circuits with one switch}

The first simulation case study refers to an electrical circuit composed of RLC elements and one switch. The schematic diagram of the considered circuit is shown is Fig. 5.

This circuit is simulated within the EMTP-RV simulation environment considering both an ideal model and a discrete-time model for the switch. As previously mentioned, the backward Euler method was used for the numerical integration with a time step $\Delta t=4 \mu \mathrm{s}$.

For the circuit shown in Fig. 5, the nodal admittance matrices are formed for the cases where the switch is represented by (i)FAMNM, (ii) ideal switch in 'on' case, and (iii) ideal switch in 'off' case. Then, according to the proposed method, the objective function (15) is determined. In order to calculate the error and losses functions, the following switching transition is considered: the switch is in open position and it is closed at $t=10 \mathrm{~ms}$. Then, it is opened again at $t=20 \mathrm{~ms}$. For all values of $G_{s}$ (i.e., $0 \leq G_{s} \leq 1$ ), Eqs. (15), (20) and (24) are calculated. The objective function together with the corresponding error and losses functions are shown in Fig. 6. As it can be clearly observed, all the three functions have their minimum when $G_{s}$ is equal to 0.11 .

The second simulation example refers to a network as shown in Fig. 2, which includes a single-conductor transmission line.

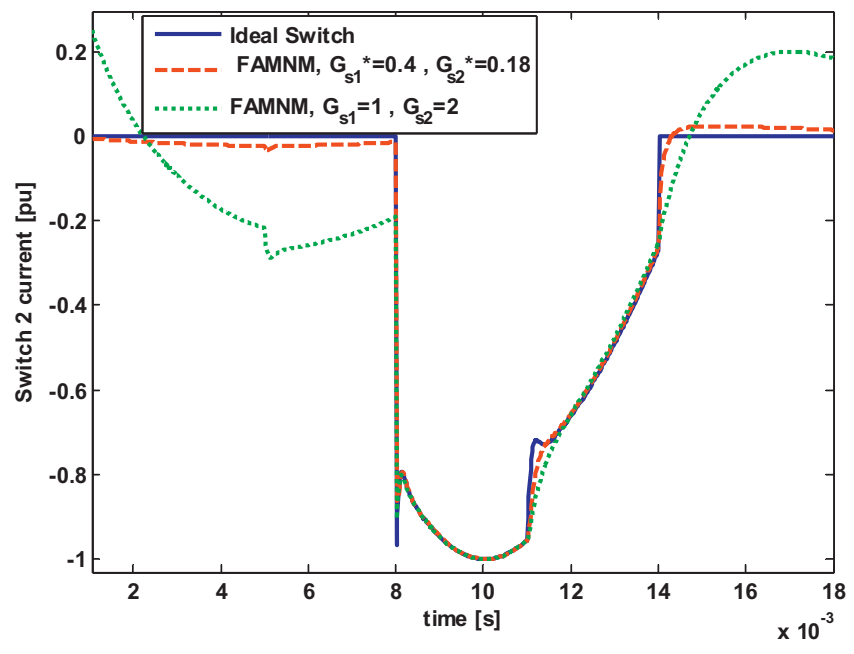

Fig. 12. Time-domain simulated waveforms for switch \#2 current for the optimal values of $G_{s 1}, G_{s 2}$ for the circuit composed of RLC elements and two switches. 
By applying the same procedure for $0 \leq G_{S} \leq 1$, the objective, error, and losses functions are calculated (see Fig. 7). As it is shown on Fig. 7, these three functions exhibit the same behavior as for the previous case, with a common minimum occurring for a value of $G_{s}$ equal to 0.01 .

With reference to the second test case, Fig. 8 illustrates the time-domain simulations of the voltage at the end of the line for different values of $G_{S}$ including the optimal value previously identified $\left(G_{s}^{*}=0.01\right)$. It can be seen that the simulations obtained using the optimum value for the conductance are in agreement with those obtained using EMTP-RV.

Figs. 6 and 7 show that the proposed objective function could be utilized as an efficient tool to find the optimum $G_{s}$ value without performing any off-line benchmark simulations, as the effect of $G_{s}$ on the adopted system model could be correctly predicted.

\subsection{Circuits with two switches}

In order to validate the performance of the proposed method, additional investigations have been done for the case of networks with two switches. To this end, the simulation example refers to an RLC circuit including two switches. The schematic diagram of the considered circuit is shown is Fig. 9.

According to the proposed method, for each switch, a dedicated $G_{s}$ value is considered and Eqs. (15), (20), and (24) are formed to find the objective, error and losses functions. Fig. 10 shows the objective function. As it is shown on this figure, the minimum values correspond to $G_{s 1}^{*}=0.4$ and $G_{s 2}^{*}=0.18$.

In Figs. 11 and 12, the time-domain simulations of the voltage and current of switch \#2 of Fig. 9 show that a very good match is achieved between the results of the FAMNM representation with optimal conductance value and the ideal representation.

\section{Conclusions}

In this paper, a method to find the optimum value of discretetime switch conductance has been proposed. As known, the value of this parameter should be chosen in a way to minimize the errors introduced by the approximate representation of the discrete-time switch model.

The proposed method is based on the minimization of the Euclidian distance between the eigenvalues of the FAMNM network admittance matrix and those associated with the admittance matrices of the reference networks corresponding to all possible switches permutations. Additionally, the proposed method avoids the use of trial-and-error process typically required when discrete-time switch conductances need to be addressed in FAMNM approach.

To prove the correctness of the proposed method, a comparison between the proposed metric and specific defined error functions has been presented and discussed. By making reference to two different categories of test cases, namely (i) circuits with a single switch and (ii) circuits with multiple switches, the proposed method has been proven to be correct in identifying the optimal conductance value of the discrete-time switch model. It is also shown that the proposed method minimizes simultaneously the differences with reference-model current/voltage waveforms, and losses on the discrete-time switch conductance.
Without the need of performing off-line benchmark simulations, the proposed method can represent a powerful solution for the representation of switches within real-time simulation platforms that rely on the use of the FAMNM (i.e., FPGAs).

\section{Acknowledgment}

The paper takes into account the comments received during the presentation of a preliminary version at the 10th International Conference on Power Systems Transients (IPST 2013), Vancouver, Canada, July, 2013. This work was supported by the Swiss National Science Foundation (Project No. 200021-146414, "Power Networks Fault Location based on Electronic Emulation").

\section{References}

[1] P. Pejovic, D. Maksimovic, A method for fast time-domain simulation of networks with switches, IEEE Trans. Power Electron. 9 (4) (1994) 449-456.

[2] M. Matar, R. Iravani, An FPGA-based real-time digital simulator for power electronic systems, in: International Conference on Power Systems Transients (IPST'07), Lyon, France, June, 2007.

[3] H.J. Wu, W.S. Feng, Efficient simulation of switched networks using reduced unification matrix, IEEE Trans. Power Electron. 14 (3) (1999) 481-494.

[4] H.W. Dommel, EMTP Theory Book, B.P.A., 1986, August.

[5] M. Matar, R. Iravani, FPGA implementation of the power electronic converter model for real-time simulation of electromagnetic transients, IEEE Trans. Power Delivery 25 (2) (2010) 852-860.

[6] S.Y.R. Hui, K.K. Fung, Fast decoupled simulation of large power electronic systems using new two-port companion link models, IEEE Trans. Power Electron. 12 (3) (1997) 462-473.

[7] S.Y.R. Hui, S. Morrall, Generalised associated discrete circuit model for switching devices, IEE Proc. Sci. Meas. Technol. 141 (1) (1994) 57-64.

[8] S.Y.R. Hui, C. Christopoulos, A discrete approach to the modeling of power electronic switching networks, IEEE Trans. Power Electron. 5 (4) (1990) 398-403.

[9] C. Dufour, S. Cense, T. Ould-Bachir, L. Grégoire, J. Bélanger, General-purpose reconfigurable low-latency electric circuit and motor drive solver on FPGA, in: 38th Annual Conference on IEEE Industrial Electronics Society - IECON 2012, Montréal, Canada, October, 2012.

[10] T. Maguire, J. Giesbrecht, Small time-step ( $<2 \mu \mathrm{sec}$ ) VSC model for the real time digital simulator, in: International Conference on Power Systems Transients (IPST'05), Montreal, Canada, 2005.

[11] R. Razzaghi, C. Foti, M. Paolone, F. Rachidi, A novel method for the optima parameter selection of discrete-time switch model, in: International Conference Power Systems Transients (IPST2013), Vancouver, Canada, June, 2013.

[12] J. Mahseredjian, Computation of power system transients: overview and challenges, in: IEEE Power Engineering Society General Meeting, Florida, USA, 2007, June.

[13] C.A. Thompson, A Study of Numerical Integration Techniques for Use in the Companion Circuit Method of Transient Circuit Analysis. TR-EE 92-17, School of Electrical Engineering, Purdue University, 1992.

[14] T. Noda, K. Takenaka, T. Inoue, Numerical integration by the 2 -stage diagonally implicit Runge-Kutta method for electromagnetic transient simulations, IEEE Trans. Power Delivery 24 (1) (2009) 390-399.

[15] J. Mahseredjian, S. Lefebvre, X.-D. Do, A new method for time-domain modeling of nonlinear circuits in large linear networks, in: Proceedings of the 11th Power Systems Computation Conference PSCC, August, 1993.

[16] J. Mahseredjian, S. Dennetière, L. Dubé, B. Khodabakhchian, L. Gérin-Lajoie, On a new approach for the simulation of transients in power systems, Electric Power Syst. Res. 77 (11) (2007) 1514-1520.

[17] L. Chua, P. Lin, Computer-Aided Analysis of Electronic Circuits, Prentice Hall, New York, 1975.

[18] H.W. Dommel, Digital computer solution of electromagnetic transients in single- and multiphase networks, IEEE Trans. Power Appar. Syst. PAS-88 (1969) 388-399.

[19] J. Arrillaga, N.R. Watson, Power Systems Electromagnetic Transients Simulation, Institution of Engineering and Technology, 2002.

[20] L. Bergeron, Du coupde bélier en hydraulique au coupde foudre en electricité, Dunod, Paris, France, 1949.

[21] J.R. Marti, Accurate modeling of frequency-dependent transmission lines in electromagnetic transient simulations, IEEE Trans. Power Appar. Syst. 101 (1) (1982) 147-157. 